\title{
Infestation of Micromycetes on the Flowers and Seeds of Rhododendron sp.
}

\author{
Maria Kowalik, Klaudia Duda-Franiak and Małgorzata Rymarczyk \\ Department of Plant Protection, Faculty of Biotechnology and Horticulture, University of Agriculture in Krakow (UAK), Al. 29 \\ Listopada 54, Kraków 31-425, Poland
}

\begin{abstract}
The study of micromycetes infestation on flower buds, flower petals and seeds from 10 taxa of Rhododendron sp. was conducted during 2010-2012. Mycological analysis was comprised of 1,500 specimens of buds and petals and 500 seeds of Rhododendron sp.. It was shown that the necrosis and dieback of buds were caused by complex micromycetes (43 species), with dominants Pestalotiopsis sydowiana, Alternaria alternata, Truncatella truncata and Epicoccum nigrum. Watery brown spots on the flower petals, resulting in the dieback of flowers, were caused by 38 species, including the most common P. sydowiana, A. alternata and Trichoderma viride. The seeds were contaminated by 18 species, and in addition to the above, the following species were associated: Oidiodendron tennuissimum, Davidiella macrocarpa and Phoma leveillei. The results of the mycological analysis confirmed the diversity spectrum of micromycetes species that inhabit the infested Rhododendron buds, flowers and seeds. The research revealed which taxa attracted the largest number of colonies and species of fungi.
\end{abstract}

Key words: Rhododendron, flower buds, flower petals, seeds, fungi, infestation, health status.

\section{Introduction}

Rhododendron sp. is one of the most attractive plants in contemporary gardens. The selective breeding of Rhododendrons takes into consideration not only the value of flowers, the decorativeness of leaves, frost resistance, tolerance to adverse climate and soil conditions, but also the low susceptibility to diseases caused by biotic factors, including filamentous fungal micromycetes.

The health evaluation of interspecific evergreen Rhododendron hybrids may give a basis to the exclusion of sensitive taxa from further breeding and indicate which taxa possess flower buds, flowers and seeds that are slightly susceptible to infection by micromycetes [1-5].

The aim of the study was to compare micromycetes biodiversity inhabiting the flowers and seeds of newly grown Rhododendron sp. hybrids and also diagnose

Corresponding author: Maria Kowalik, professor, research fields: mycology, phytopathology and plant protection. E-mail: m.kowalik@ogr.ur.krakow.pl. the hybrids' susceptibility to colonization by pathogenic and saprophytic micromycetes species.

\section{Materials and Methods}

The research was carried out during 2010-2012 with the collection of ornamental plants from the Faculty of Biotechnology and Horticulture at the University of Agriculture in Krakow. The mycological assessment consisted of nine Rhododendron sp. evergreen Rhododendron interspecific hybrids, which were obtained from the selective breeding in hybridization of three East Asian species-R. aureum, $R$. brachycarpum and $R$. purdomii, with the "Catherine van Tol" cultivar from the $R$. catawbiense group, "Koichiro Wada" from R. yakushimanum and the $R$. purdomii species (Table 1). The study was carried out on 10 individual shrubs of each taxon, with the age of the shrubs being 11-13 years.

Dead flower buds were collected in October 2010-2012 and infested flowers were collected during the flowering period (from April to June). Seeds were collected once in November 2012. 
Table 1 Details of studied evergreen Rhododendron sp. taxa.

\begin{tabular}{|c|c|c|}
\hline \multicolumn{2}{|c|}{ Hybrids } & \multirow{2}{*}{ Color of flower } \\
\hline Mother plants & Male plants & \\
\hline R. aureum & R. brachycarpum & Creamy-white \\
\hline R. aureum & R. catawbiense "Catherine van Tol" & Pink \\
\hline R. aureum & R. yakushimanum "Koichiro Wada" & Creamy-white \\
\hline R. brachycarpum & R. brachycarpum & White and pink \\
\hline R. brachycarpum & R. purdomii & White and pink \\
\hline R. purdomii & R. yakushimanum "Koichiro Wada" & Pink \\
\hline R. yakushimanum "Koichiro Wada" & R. aureum & Creamy-white \\
\hline R. yakushimanum "Koichiro Wada" & R. brachycarpum & Pink \\
\hline R. yakushimanum "Koichiro Wada" & R. catawbiense "Catherine van Tol" & Pink \\
\hline R. purdomii & R. purdomii & Pink \\
\hline
\end{tabular}

Fifteen hundred pieces of buds, 1,500 pieces of flower petals and 500 seeds were allocated for mycological analysis. Fragments of buds and petals (taken from the border of living and dead tissue) and seeds were disinfected in $70 \%$ ethanol and then placed in Petri dishes with a solidified Difco ${ }^{\circledR}$ potato dextrose agar (PDA) medium. Micromycetes isolation and cultivation were conducted according to standard methods applied in mycology [5].

The keys used for the taxonomic identification of the mycobiota were these aspects by the following authors: Guba [6], Domsch et al. [7], Sutton [8], Sivanesan [9] and Ellis, M. B., and Ellis, J. P [10]. The basis of classification was the system by Kirk et al. [11], and the authors' descriptions of fungal species names were verified according to Index Fungorum [12].

On the basis of micromycetes specification and considering the share of individual species within the total fungi community, they were classified into three groups, namely, dominants (constituting $>5 \%$ of the entire community), influents (1\%-5\%) and accessory fungi $(<1 \%)$, according to Kowalik [13].

\section{Results and Discussion}

Sixty-five filamentous fungal micromycetes were found on the infected buds, petals and seeds of 10 taxa of Rhododendron. The entire community consisted of 1,934 colonies (Tables 2-4).

The symptoms of infestations on flower buds were characterized by necrosis, which led to browning and bud dieback. Nine hundred and sixty micromycetes colonies comprising 43 species from 29 genera were isolated from infested flower buds. The most common species were Pestalotiopsis sydowiana (constituting $29.49 \%$ of the entire community), Alternaria alternata (19.58\%), Truncatella truncata (10.10\%) and Epicoccum nigrum (5.31\%). These micromycetes were classified to the group of dominants. The following species were isolated in large numbers: Broomella acuta, Isaria fumorosea, Paraphoma chrysanthemicola, Phialophora asteris, P. cyclaminis, Phoma eupyrena, Ph. pinodella, Trichoderma viride and Umbelopsis isabellina. These species were classified to the group of influents. The other 31 species were classified into the accessory group.

The number of micromycetes colonies inhabiting the flower buds increased in the consecutive years of the study. The most fungi colonies (391 isolates) were isolated in 2012, representing 25 species. The fewest fungi colonies were isolated in 2010 , at which time the flower buds were inhabited by 275 colonies and 28 species of fungi (Table 2).

Watery brown spots on the flower petals, resulting in the dieback of flowers, were caused by 38 species of micromycetes within 25 genera. Eight hundred and forty-five colonies of fungi were isolated from the infected petals, including the dominants $P$. sydowiana, $A$. alternata and T. viride (56.69\% of the entire community). 
Table 2 Isolation frequency of fungi isolated from the infested buds of evergreen Rhododendron.

\begin{tabular}{|c|c|c|c|c|}
\hline \multirow{2}{*}{ Fungus } & \multicolumn{3}{|c|}{ Number of fungi colonies in years } & \multirow{2}{*}{$\begin{array}{l}\text { Percentage } \\
(\%)\end{array}$} \\
\hline & 2010 & 2011 & 2012 & \\
\hline Alternaria alternata (Fr.) Keissl. & 31 & 49 & 108 & 19.58 \\
\hline Alternaria tenuissima (Kunze) Wiltshire & 2 & - & - & 0.21 \\
\hline Aspergillus brasiliensis Varga, Frisvad \& Samson & 2 & 1 & - & 0.31 \\
\hline Aspergillus ustus (Bainier) Thom \& Church & 1 & - & - & 0.10 \\
\hline Aspergillus versicolor (Vuill.) Tirab. & 4 & 3 & - & 0.73 \\
\hline Boeremia exigua (Desm.) Aveskamp, Gruyter \& Verkley & 1 & - & 6 & 0.73 \\
\hline Botrytis cinerea Pers. & 1 & - & 6 & 0.73 \\
\hline Broomella acuta Shoemaker \& E. Müll. & - & - & 12 & 1.25 \\
\hline Calonectria morganii Crous, Alfenas \& M.J. Wingf. & - & - & 5 & 0.52 \\
\hline Cladosporium cladosporioides (Fresen.) G.A. de Vries & - & - & 5 & 0.52 \\
\hline Cladosporium herbarum (Pers.) Link & 2 & - & - & 0.21 \\
\hline Epicoccum nigrum Link & 15 & 13 & 23 & 5.31 \\
\hline Fusarium poae (Peck) Wollenw. & 4 & 2 & - & 0.63 \\
\hline Isaria fumosorosea Wize & - & - & 34 & 3.54 \\
\hline Khuskia oryzae K.J. Huds & - & - & 8 & 0.83 \\
\hline Mammaria echinobotryoides Ces. & 4 & 3 & - & 0.73 \\
\hline Monodictys castaneae (Wallr.) S. Hughes & - & - & 1 & 0.10 \\
\hline Mortierella alpina Peyronel & - & - & 2 & 0.21 \\
\hline Mortierella bainieri Costantin & 1 & 1 & - & 0.21 \\
\hline Mortierella hyalina (Harz) W. Gams & - & 1 & - & 0.10 \\
\hline Mucor racemosus f. sphaerosporus (Hagem) Schipper & 2 & - & 1 & 0.31 \\
\hline Myrothecium roridum Tode & - & - & 2 & 0.21 \\
\hline Oidiodendron tenuissimum (Peck) S. Hughes & - & - & 4 & 0.42 \\
\hline Paraphoma chrysanthemicola (Hollós) Gruyter, Aveskamp \& Verkley & 6 & 10 & 1 & 1.77 \\
\hline Penicillium expansum Link & 3 & 2 & - & 0.52 \\
\hline Penicillium waksmanii K.M. Zaleski & 2 & 3 & - & 0.52 \\
\hline Pestalotiopsis sydowiana (Bres.) B. Sutton & 109 & 89 & 85 & 29.49 \\
\hline Phialophora asteris (Dowson) Burge \& I. Isaac & 13 & 21 & 2 & 3.75 \\
\hline Phialophora cinerescens (Wollenw.) J.F.H. Beyma & - & 2 & - & 0.21 \\
\hline Phialophora cyclaminis J.F.H. Beyma & 8 & 17 & 15 & 4.17 \\
\hline Phoma eupyrena Sacc. & 3 & 3 & 8 & 1.46 \\
\hline Phoma leveillei Boerema \& G.J. Bollen & - & - & 8 & 0.83 \\
\hline Phoma pinodella (L.K. Jones) Morgan-Jones \& K.B. Burch & & 3 & 19 & 2.29 \\
\hline $\begin{array}{l}\text { Pleurostomophora richardsiae (Nannf.) L. Mostert, } \\
\text { W. Gams \& Crous }\end{array}$ & 2 & 1 & - & 0.31 \\
\hline Sordaria fimicola (Roberge ex Desm.) Ces. \& De Not. & - & - & 1 & 0.10 \\
\hline Talaromyces wortmannii C.R. Benj. & 3 & 6 & - & 0.94 \\
\hline Trichoderma koningii Oudem. & 1 & - & - & 0.10 \\
\hline Trichoderma pseudokoningii Rifai & 1 & 1 & - & 0.21 \\
\hline Trichoderma viride Pers. & 13 & 17 & - & 3.13 \\
\hline Truncatella truncata (Lév.) Steyaert & 36 & 42 & 19 & 10.10 \\
\hline Ulocladium consortiale (Thüm.) E.G. Simmons & - & - & 2 & 0.21 \\
\hline Umbelopsis isabellina (Oudem.) W. Gams & 3 & 4 & 14 & 2.19 \\
\hline Umbelopsis nana (Linnem.) Arx & 2 & - & - & 0.21 \\
\hline Total & 275 & 294 & 391 & 100.00 \\
\hline
\end{tabular}


Table 3 Isolation frequency of fungi isolated from the infested petals of evergreen Rhododendron.

\begin{tabular}{|c|c|c|c|c|}
\hline \multirow{2}{*}{ Fungus } & \multicolumn{3}{|c|}{ Number of fungi colonies in years } & \multirow{2}{*}{$\begin{array}{l}\text { Percentage } \\
(\%)\end{array}$} \\
\hline & 2010 & 2011 & 2012 & \\
\hline Absidia spinosa Lendn. & - & - & 3 & 0.36 \\
\hline Acremonium rutilum $\mathrm{W}$. Gams & 1 & 2 & 5 & 0.95 \\
\hline Alternaria alternata (Fr.) Keissl. & 76 & 98 & 28 & 23.91 \\
\hline Alternaria tenuissima (Kunze) Wiltshire & - & - & 3 & 0.36 \\
\hline Arthrinium phaeospermum (Corda) M.B. Ellis & - & 1 & - & 0.12 \\
\hline Aspergillus brasiliensis Varga, Frisvad \& Samson & - & - & 15 & 1.78 \\
\hline Aspergillus ustus (Bainier) Thom \& Church & - & - & 1 & 0.12 \\
\hline Aspergillus versicolor (Vuill.) Tirab. & - & - & 2 & 0.24 \\
\hline Botrytis cinerea Pers. & 2 & 6 & 3 & 1.30 \\
\hline Chaetomium funicola Cooke & 1 & 2 & 10 & 1.54 \\
\hline Chaetomium globosum Kunze & 3 & 4 & 1 & 0.95 \\
\hline Cladosporium cladosporioides (Fresen.) G.A. de Vries & - & 1 & - & 0.12 \\
\hline Coleophoma rhododendri Syd. & - & - & 1 & 0.12 \\
\hline Epicoccum nigrum Link & 16 & 23 & - & 4.62 \\
\hline Fusarium culmorum (Wm.G. Sm.) Sacc. & - & 7 & - & 0.83 \\
\hline Fusarium oxysporum Schltdl. & - & - & 1 & 0.12 \\
\hline Gibberella avenacea R.J. Cook & 4 & 9 & - & 1.54 \\
\hline Humicola fuscoatra Traaen & 2 & 3 & - & 0.59 \\
\hline Mortierella alpina Peyronel & - & 3 & - & 0.36 \\
\hline Mortierella hyalina (Harz) W. Gams & 6 & 3 & - & 1.07 \\
\hline Mucor hiemalis Wehmer & - & - & 4 & 0.47 \\
\hline Penicillium citrinum Thom & - & - & 10 & 1.18 \\
\hline Pestalotiopsis sydowiana (Bres.) B. Sutton & 78 & 91 & 65 & 27.69 \\
\hline Phialophora cyclaminis J.F.H. Beyma & - & - & 38 & 4.50 \\
\hline Phoma eupyrena Sacc. & 7 & 1 & 16 & 2.84 \\
\hline Phoma laundoniae Boerema \& Gruyter & - & - & 8 & 0.95 \\
\hline Phoma leveillei Boerema \& G.J. Bollen & - & 1 & 9 & 1.18 \\
\hline Phoma pinodella (L.K. Jones) Morgan-Jones \& K.B. Burch & - & 2 & 2 & 0.47 \\
\hline Phoma putaminum Speg. & - & - & 2 & 0.24 \\
\hline Sclerotinia sclerotiorum (Lib.) de Bary & 3 & 12 & - & 1.78 \\
\hline Scopulariopsis fusca Zach & - & - & 1 & 0.12 \\
\hline Tetracoccosporium paxianum Szabó & - & 1 & - & 0.12 \\
\hline Trichoderma hamatum (Bonord.) Bainier & - & - & 1 & 0.12 \\
\hline Trichoderma harzianum Rifai & - & 4 & 13 & 2.01 \\
\hline Trichoderma koningii Oudem. & 10 & 12 & 9 & 3.67 \\
\hline Trichoderma viride Pers. & 2 & 5 & 36 & 5.09 \\
\hline Truncatella truncata (Lév.) Steyaert & 12 & 15 & 3 & 3.55 \\
\hline Umbelopsis isabellina (Oudem.) W. Gams & 7 & 18 & 1 & 3.08 \\
\hline Total & 230 & 324 & 291 & 100.00 \\
\hline
\end{tabular}

The influents group included Aspergillus brasilensis, Botrytis cinerea, Chaetomium funicola, E. nigrum, Gibberella avenacea, Mortierella hyalina, Penicillium citrinum, Ph. cyclaminis, Ph. eupyrena, Ph. leveillei, Sclerotinia sclerotiorum, Trichoderma harzianum, T. koningii, T. truncata and U. isabellina.
Colonies of these micromycetes constituted more than $32 \%$ of the total isolated fungi.

The most micromycetes in the number of 324 colonies and 24 species were isolated from infected flowers in 2011. The lowest micromycetes was isolated from infected flowers in 2010, inhabiting 16 
Table 4 Isolation frequency of fungi isolated from the seeds of evergreen Rhododendron.

\begin{tabular}{lll}
\hline Fungus & Number of fungi colonies in 2012 & $\begin{array}{l}\text { Percentage } \\
(\%)\end{array}$ \\
\hline Alternaria alternata (Fr.) Keissl. & 29 & 22.48 \\
Broomella acuta Shoemaker \& E. Müll. & 1 & 0.78 \\
Davidiella macrocarpa Crous, K. Schub. \& U. Braun & 10 & 7.75 \\
Epicoccum nigrum Link & 13 & 10.08 \\
Haematonectria haematococca (Berk. \& Broome) Samuels \& Rossman & 6 & 4.65 \\
Isaria fumosorosea Wize & 1 & 0.78 \\
Khuskia oryzae K.J. Huds & 2 & 1.55 \\
Mortierella alpina Peyronel & 3 & 2.33 \\
Oidiodendron tenuissimum (Peck) S. Hughes & 11 & 8.53 \\
Pestalotiopsis sydowiana (Bres.) B. Sutton & 20 & 15.50 \\
Phialophora asteris (Dowson) Burge \& I. Isaac & 1 & 0.78 \\
Phialophora cyclaminis J.F.H. Beyma & 2 & 1.55 \\
Phoma eupyrena Sacc. & 1 & 0.78 \\
Phoma leveillei Boerema \& G.J. Bollen & 8 & 6.20 \\
Sordaria fimicola Roberge ex Desm.) Ces. \& De Not. & 1 & 0.78 \\
Stachybotrys elegans (Pidopl.) W. Gams & 4 & 3.10 \\
Truncatella truncata (Lév.) Steyaert & 10 & 7.75 \\
Umbelopsis isabellina (Oudem.) W. Gams & 6 & 4.65 \\
\hline Total & 129 & 100.00 \\
\hline
\end{tabular}

species and representing 230 colonies (Table 3 ).

The seeds were contaminated by 18 micromycetes species. Species found in large numbers were $A$. alternata, P. sydowiana, E. nigrum, Oidiodendron tenuissimum, Davidiella macrocarpa and $P h$. leveillei. These dominants constituted more than $72 \%$ of the fungi colonies inhabiting seeds (Table 4).

The authors found differences among the taxa when comparing the number of micromycetes colonies and species on the infested organs of 10 Rhododendron taxa. From 60 to 130 colonies and from 7 to 21 species were isolated from dead flower buds. From 30 to 127 colonies and from 8 to 18 species were obtained from infected flowers. From 7 to 21 colonies and from 1 to 7 fungi species were obtained from the seeds (Tables 5-7).

The greatest number of fungi colonies was isolated from the buds flowers of hybrid obtained from $R$. brachycarpum $\times R$. brachycarpum. The dominants among the 18 species were $A$. alternata, $P$. sydowiana, Ph. asteris and Ph. cyclaminis. The lowest number of fungi colonies in each year of the analysis was reported on $R$. purdomii and the lowest number of species (seven) was reported on $R$. yakushimanum "Koichiro Wada" $\times R$. aureum. The most numerous fungi species (21) were isolated from the hybrid $R$. yakushimanum "Koichiro Wada" $\times$ R. catawbiense "Catherine van Tol" (Table 5).

The most numerous fungi species were isolated from the petals of $R$. aureum $\times R$. catawbiense "Catherine van Tol". Among the 12 species found, the following dominated: A. alternata, $P$. sydowiana, $S$. sclerotiorum, E. nigrum and $P$. citrinum. Only 30 micromycetes colonies belonging to 11 species were isolated from the flowers of the hybrid obtained from $R$. brachycarpum $\times R$. brachycarpum hybrid, which was not correlated with the high number of fungi colonies isolated from the flower buds. The most abundant in terms of micromycetes species diversity were the flowers of hybrid $R$. yakushimanum "Koichiro Wada" $\times R$. brachycarpum, of which 18 fungi species representing only 60 colonies were isolated. 
Table 5 Isolation frequency of fungi isolated from the infested flower buds of particular evergreen Rhododendron taxa.

\begin{tabular}{|c|c|c|c|c|c|c|c|c|c|c|c|}
\hline \multirow[b]{2}{*}{ Fungi taxon } & \multicolumn{11}{|c|}{ Number of fungi colonies } \\
\hline & $\begin{array}{l}R \text {. aureum } \times R \text {. } \\
\text { brachycarpum }\end{array}$ & $\begin{array}{l}\text { R. aureum } \times R \text {. } \\
\text { catawbiense }\end{array}$ & $\begin{array}{l}\text { R. aureum } \times R \text {. } \\
\text { yakushimanum }\end{array}$ & $\begin{array}{l}R \text {. brachycarpum } \times \\
R \text {. brachycarpum } \\
\end{array}$ & $\begin{array}{l}\text { R. brachycarpum } \\
\times \text { R. purdomii } \\
\end{array}$ & $\begin{array}{l}\text { R. purdomii } \times R \text {. } \\
\text { yakushimanum }\end{array}$ & $\begin{array}{l}\text { R. yakushimanum } \\
\times \text { R. aureum } \\
\end{array}$ & $\begin{array}{l}\text { R. yakushimanum } \times \\
\text { R. brachycarpum }\end{array}$ & $\begin{array}{l}\text { R. yakushimanum } \\
\times R \text {. catawbiense }\end{array}$ & $\begin{array}{l}R . \\
\text { purdomii }\end{array}$ & Total \\
\hline A. alternata & 35 & 17 & 12 & 27 & 31 & 13 & 1 & 34 & 11 & 7 & 188 \\
\hline A. brasiliensis & - & - & - & - & - & 3 & - & - & - & - & 3 \\
\hline A. ustus & - & - & - & - & - & - & - & 1 & - & - & 1 \\
\hline A. versicolor & - & - & - & - & - & 1 & - & - & 6 & - & 7 \\
\hline B. cinerea & 3 & 1 & - & - & 2 & - & - & - & - & 1 & 7 \\
\hline B. acuta & - & 1 & 11 & - & - & - & - & - & - & - & 12 \\
\hline C. morganii & - & - & - & 5 & - & - & - & - & - & - & 5 \\
\hline C. cladosporioides & - & - & - & - & - & 5 & - & - & - & - & 5 \\
\hline C. herbarum & - & - & - & - & - & 2 & - & - & - & - & 2 \\
\hline E. nigrum & 10 & 4 & 7 & 2 & 3 & 4 & - & - & 20 & 1 & 51 \\
\hline F. poae & - & 4 & - & 2 & - & - & - & - & - & - & 6 \\
\hline M. echinobotryoides & 3 & 4 & - & - & - & - & - & - & - & - & 7 \\
\hline M. castaneae & - & - & - & - & 1 & - & - & - & - & - & 1 \\
\hline M. alpina & 1 & - & - & - & - & - & 1 & - & - & & 2 \\
\hline M. bainieri & & 1 & - & - & - & - & - & - & 1 & - & 2 \\
\hline M. hyalina & - & - & - & - & - & - & - & - & 1 & - & 1 \\
\hline M. racemosus & - & - & - & 1 & - & - & - & 2 & - & - & 3 \\
\hline M. roridum & - & - & - & - & - & - & - & 2 & - & - & 2 \\
\hline O. tenuissimum & - & - & - & - & - & - & - & 1 & - & 3 & 4 \\
\hline P. chrysanthemicola & 9 & - & - & - & - & 8 & - & - & - & - & 17 \\
\hline P. expansum & - & - & - & - & - & - & - & - & 5 & - & 5 \\
\hline P. waksmanii & 1 & - & - & - & - & - & - & - & 4 & - & 5 \\
\hline P. sydowiana & 37 & 25 & 41 & 12 & 18 & 36 & 58 & 22 & 4 & 30 & 283 \\
\hline Ph. asteris & - & - & 1 & 13 & 16 & - & - & - & 5 & 1 & 36 \\
\hline Ph. cinerescens & - & - & - & 1 & - & - & - & - & 1 & - & 2 \\
\hline
\end{tabular}


(Table 5 continued)

\begin{tabular}{|c|c|c|c|c|c|c|c|c|c|c|c|}
\hline \multirow[b]{2}{*}{ Fungi taxon } & \multicolumn{11}{|c|}{ Number of fungi colonies } \\
\hline & $\begin{array}{l}R . \text { aureum } \times R \text {. } \\
\text { brachycarpum }\end{array}$ & $\begin{array}{l}\text { R. aureum } \times R \text {. } \\
\text { catawbiense }\end{array}$ & $\begin{array}{l}\text { R. aureum } \times R \text {. } \\
\text { yakushimanum }\end{array}$ & $\begin{array}{l}\text { R. brachycarpum } \times \\
\text { R. brachycarpum } \\
\end{array}$ & $\begin{array}{l}\text { R. brachycarpum } \\
\times \text { R. purdomii } \\
\end{array}$ & $\begin{array}{l}\text { R. purdomii } \times R \text {. } \\
\text { yakushimanum }\end{array}$ & $\begin{array}{l}\text { R. yakushimanum } \\
\times \text { R. aureum } \\
\end{array}$ & $\begin{array}{l}\text { R. yakushimanum } \times \\
\text { R. brachycarpum }\end{array}$ & $\begin{array}{l}\text { R. yakushimanum } \\
\times \text { R. catawbiense } \\
\end{array}$ & $\begin{array}{l}R . \\
\text { purdomii }\end{array}$ & Total \\
\hline Ph. cyclaminis & - & 1 & 2 & 21 & 2 & - & 3 & 4 & 7 & - & 40 \\
\hline Ph. leveillei & - & 5 & - & - & 1 & 1 & - & - & 1 & - & 8 \\
\hline Ph. pinodella & - & 2 & 2 & 11 & - & - & - & - & - & 7 & 22 \\
\hline P. richardsiae & 1 & - & - & 1 & - & - & - & - & 1 & - & 3 \\
\hline T. wortmannii & - & - & - & 9 & - & - & - & - & - & - & 9 \\
\hline T. koningii & - & - & - & - & - & - & - & - & 1 & - & 1 \\
\hline T. pseudokoningii & - & - & - & - & - & 2 & - & - & - & - & 2 \\
\hline T. viride & - & - & - & - & - & 8 & 10 & - & 12 & - & 30 \\
\hline T. truncata & 15 & 7 & 22 & 7 & 10 & 4 & 11 & 20 & - & 1 & 97 \\
\hline U. consortiale & - & - & - & - & - & - & - & - & 1 & 1 & 2 \\
\hline U. isabellina & - & - & - & - & - & - & 15 & 5 & 1 & - & 21 \\
\hline $\begin{array}{l}\text { Total colonies } \\
\text { /species }\end{array}$ & $\begin{array}{l}117 / \\
11\end{array}$ & $\begin{array}{l}72 / \\
12\end{array}$ & $\begin{array}{l}103 / \\
10\end{array}$ & $\begin{array}{l}130 / \\
18\end{array}$ & $\begin{array}{l}86 / \\
10\end{array}$ & $\begin{array}{l}87 / \\
12\end{array}$ & $\begin{array}{l}99 / \\
7\end{array}$ & $\begin{array}{l}101 / \\
11\end{array}$ & $\begin{array}{l}105 / \\
21\end{array}$ & $\begin{array}{l}60 / \\
11 \\
\end{array}$ & $\begin{array}{l}960 / \\
43\end{array}$ \\
\hline
\end{tabular}

Table 6 Isolation frequency of fungi isolated from the infested petals of particular evergreen Rhododendron taxa.

\begin{tabular}{|c|c|c|c|c|c|c|c|c|c|c|c|}
\hline \multirow[b]{2}{*}{ Fungi taxon } & \multicolumn{11}{|c|}{ Number of fungi colonies } \\
\hline & $\begin{array}{l}\text { R. aureum } \times R \text {. } \\
\text { brachycarpum }\end{array}$ & $\begin{array}{l}\text { R. aureum } \times R \text {. } \\
\text { catawbiense }\end{array}$ & $\begin{array}{l}R \text {. aureum } \times R . \\
\text { yakushimanum }\end{array}$ & $\begin{array}{l}\text { R. brachycarpum } \times \\
\text { R. brachycarpum }\end{array}$ & $\begin{array}{l}\text { R. brachycarpum } \\
\times \text { R. purdomii } \\
\end{array}$ & $\begin{array}{l}\text { R. purdomii } \times R \text {. } \\
\text { yakushimanum }\end{array}$ & $\begin{array}{l}\text { R. yakushimanum } \\
\times \text { R. aureum } \\
\end{array}$ & $\begin{array}{l}\text { R. yakushimanum } \times \\
\text { R. brachycarpum }\end{array}$ & $\begin{array}{l}\text { R. yakushimanum } \\
\times R \text {. catawbiense } \\
\end{array}$ & $\begin{array}{l}R . \\
\text { purdomii }\end{array}$ & Total \\
\hline A. spinosa & - & - & - & 1 & - & 2 & - & - & - & - & 3 \\
\hline A. rutilum & - & 2 & 1 & - & - & - & - & 5 & - & - & 8 \\
\hline A. alternata & 36 & 43 & 29 & 8 & 11 & 10 & 14 & 8 & 28 & 15 & 202 \\
\hline A tennuissima & - & - & - & - & & 3 & - & - & - & - & 3 \\
\hline A. pheospermum & - & - & - & - & 1 & - & - & - & - & - & 1 \\
\hline A. brasiliensis & - & - & - & - & 1 & 9 & - & - & - & 5 & 15 \\
\hline A. ustus & - & - & - & - & - & - & - & - & - & 1 & 1 \\
\hline A. versicolor & - & 1 & - & - & 1 & - & - & - & - & - & 2 \\
\hline
\end{tabular}


(Table 6 continued)

\begin{tabular}{|c|c|c|c|c|c|c|c|c|c|c|c|}
\hline \multirow[b]{2}{*}{ Fungi taxon } & \multicolumn{11}{|c|}{ Number of fungi colonies } \\
\hline & $\begin{array}{l}\text { R. aureum } \times R \text {. } \\
\text { brachycarpum }\end{array}$ & $\begin{array}{l}\text { R. aureum } \times R \text {. } \\
\text { catawbiense }\end{array}$ & $\begin{array}{l}R \text {. aureum } \times R \text {. } \\
\text { yakushimanum }\end{array}$ & $\begin{array}{l}\text { R. brachycarpum } \times \\
R . \text { brachycarpum }\end{array}$ & $\begin{array}{l}R \text {. brachycarpum } \\
\times R \text {. purdomii }\end{array}$ & $\begin{array}{l}\text { R. purdomii } \times R \text {. } \\
\text { yakushimanum }\end{array}$ & $\begin{array}{l}\text { R. yakushimanum } \\
\times R \text {. aureum }\end{array}$ & $\begin{array}{l}\text { R. yakushimanum } \times \\
\text { R. brachycarpum }\end{array}$ & $\begin{array}{l}R . \text { yakushimanum } \\
\times R \text {. catawbiense } \\
\end{array}$ & $\begin{array}{l}\text { R. } \\
\text { purdomii }\end{array}$ & Total \\
\hline B. cinerea & - & - & - & - & 1 & 3 & - & 7 & - & - & 11 \\
\hline Ch. funicola & 8 & 2 & - & 3 & - & - & - & - & - & - & 13 \\
\hline Ch. globosum & & 1 & - & - & - & 3 & 1 & 2 & 1 & - & 8 \\
\hline C. cladosporioides & - & - & - & - & - & - & - & - & 1 & - & 1 \\
\hline C. rhododendri & - & - & - & - & - & - & - & 1 & - & - & 1 \\
\hline E. nigrum & 5 & 10 & 4 & 1 & - & - & 7 & 2 & 5 & 5 & 39 \\
\hline F. oxysporum & - & - & - & - & - & - & - & - & 1 & - & 1 \\
\hline G. avenacea & - & - & 2 & - & 3 & & 6 & 2 & - & - & 13 \\
\hline H. fuscoatra & - & - & 1 & - & 1 & 1 & 1 & 1 & - & - & 5 \\
\hline M. alpina & - & - & - & - & - & - & - & 3 & - & - & 3 \\
\hline M. hyalina & - & - & - & - & 6 & - & - & 3 & - & - & 9 \\
\hline M. hiemalis & - & - & - & - & - & - & - & - & 4 & - & 4 \\
\hline P. citrinum & - & 10 & - & - & - & - & - & - & - & - & 10 \\
\hline P. sydowiana & 29 & 32 & 32 & 7 & 15 & 15 & 57 & 6 & 28 & 13 & 234 \\
\hline Ph. eupyrena & - & - & 9 & 2 & 1 & 2 & 3 & 3 & - & 4 & 24 \\
\hline Ph. laundoniae & - & - & - & - & - & - & - & - & - & 8 & 8 \\
\hline Ph. leveillei & - & - & - & 2 & 1 & 1 & 4 & 2 & - & - & 10 \\
\hline Ph. pinodella & - & - & - & - & 2 & 1 & - & - & - & 1 & 4 \\
\hline Ph. putaminum & - & - & - & - & - & - & 2 & - & - & - & 2 \\
\hline S. sclerotiorum & - & 12 & - & - & - & - & - & 3 & - & - & 15 \\
\hline S. fusca & 1 & - & - & - & - & - & - & - & - & - & 1 \\
\hline T. paxianum & - & - & 1 & - & - & - & - & - & - & - & 1 \\
\hline T. hamatum & - & - & - & 1 & - & - & - & - & - & - & 1 \\
\hline T. harzianum & - & - & 10 & - & - & - & - & - & 6 & 1 & 17 \\
\hline T. koningii & 8 & 8 & - & 3 & 6 & - & - & 3 & 1 & 2 & 31 \\
\hline T. viride & 26 & - & - & - & 4 & 2 & - & - & 11 & - & 43 \\
\hline T. truncata & - & 4 & 12 & 1 & 2 & - & 3 & 1 & 5 & 2 & 30 \\
\hline U. isabellina & 3 & 2 & - & 1 & - & - & - & 4 & 14 & 2 & 26 \\
\hline Total colonies/ & $116 /$ & $127 /$ & $106 /$ & $30 /$ & $59 /$ & $61 /$ & $113 /$ & $60 /$ & $107 /$ & $66 /$ & $845 /$ \\
\hline species & 8 & 12 & 11 & 11 & 16 & 13 & 12 & 18 & 13 & 13 & 38 \\
\hline
\end{tabular}


Table 7 Isolation frequency of fungi isolated from the seeds of particular evergreen Rhododendron taxa.

\begin{tabular}{|c|c|c|c|c|c|c|c|c|c|c|c|}
\hline \multirow[b]{2}{*}{ Fungi taxon } & \multicolumn{11}{|c|}{ Number of fungi colonies } \\
\hline & $\begin{array}{l}R \text {. aureum } \times R \text {. } \\
\text { brachycarpum }\end{array}$ & $\begin{array}{l}\text { R. aureum } \times R \text {. } \\
\text { catawbiense }\end{array}$ & $\begin{array}{l}R . \text { aureum } \times R . \\
\text { yakushimanum }\end{array}$ & $\begin{array}{l}\text { R. brachycarpum } \times \\
\text { R. brachycarpum }\end{array}$ & $\begin{array}{l}\text { R. brachycarpum } \\
\times \text { R. purdomii } \\
\end{array}$ & $\begin{array}{l}\text { R. purdomii } \times R \text {. } \\
\text { yakushimanum }\end{array}$ & $\begin{array}{l}\text { R. yakushimanum } \\
\times \text { R. aureum } \\
\end{array}$ & $\begin{array}{l}\text { R. yakushimanum } \times \\
\text { R. brachycarpum } \\
\end{array}$ & $\begin{array}{l}R \text { R. yakushimanum } \\
\times R \text {. catawbiense } \\
\end{array}$ & R. purdomii & Total \\
\hline A. alternata & 1 & 8 & 1 & 2 & 2 & 10 & - & - & 4 & 1 & 29 \\
\hline B. acuta & - & 1 & - & - & - & - & - & - & - & - & 1 \\
\hline D. macrocarpa & - & - & - & - & - & - & 10 & - & - & - & 10 \\
\hline E. nigrum & 5 & - & 2 & - & 1 & 1 & - & - & 3 & 1 & 13 \\
\hline H. haematococca & - & - & - & - & 3 & 3 & - & - & - & - & 6 \\
\hline I. fumosorosea & 1 & - & - & - & - & - & - & - & - & - & 1 \\
\hline K. oryzae & - & - & - & - & 1 & - & - & - & - & 1 & 2 \\
\hline M. alpina & - & - & - & - & - & 3 & - & - & - & - & 3 \\
\hline O. tenuissimum & - & - & - & - & - & - & - & 10 & - & 1 & 11 \\
\hline Ph. asteris & - & - & - & - & - & - & - & - & - & 1 & 1 \\
\hline Ph. cyclaminis & - & - & - & 1 & - & - & - & - & - & 1 & 2 \\
\hline Ph. eupyrena & - & 1 & - & - & - & - & - & - & - & - & 1 \\
\hline Ph. leveillei & - & - & - & - & - & - & - & 5 & 1 & 2 & 8 \\
\hline S. fimicola & - & - & - & - & 1 & - & - & - & - & - & 1 \\
\hline S. elegans & - & - & - & 1 & - & 3 & - & - & - & - & 4 \\
\hline T. truncata & - & - & 10 & - & - & - & - & - & - & - & 10 \\
\hline U. isabellina & - & 3 & - & - & - & - & - & 3 & - & - & 6 \\
\hline $\begin{array}{l}\text { Total colonies/ } \\
\text { species }\end{array}$ & $7 / 3$ & $13 / 4$ & $18 / 4$ & $7 / 4$ & $14 / 6$ & $21 / 6$ & $10 / 1$ & $18 / 3$ & $13 / 4$ & $8 / 7$ & $129 / 18$ \\
\hline
\end{tabular}


A. alternata and $P$. sydowiana inhabited the buds and flowers of all of the investigated taxa in every year.

The most numerous fungi colonies (21) were isolated from the seeds of the $R$. purdomii $\times R$. yakushimanum "Koichiro Wada" hybrid. Among the six species, A. alternata dominated. Few colonies inhabited the seeds of the $R$. aureum $\times R$. brachycarpum and $R$. brachycarpum $\times R$. brachycarpum hybrids. The seeds of $R$. purdomii were a reservoir for the largest number of micromycetes, because seven species were isolated from the seeds of this taxon in contrast to R. yakushimanum "Koichiro Wada" $\times R$. aureum seeds, which were inhabited only by D. macrocarpa (Table 7).

Shrubs of newly bred taxa of evergreen Rhododendron, growing in the collection of ornamental plants of the Faculty of Biotechnology and Horticulture at the University of Agriculture in Krakow, were found to be under pressure from saprotrophic and pathogenic fungi, which cause the dieback of buds and flowers and reduce the decorative value of the plants. The following authors have written about the health of evergreens: Farr et al. [14], Werner et al. [15], Garibaldi et al. [16], Żołna et al. [17], Czekalski and Frużyńska-Jóźwiak [1] and Kowalik [5, 13], emphasizing the diversity of micromycetes species inhabiting infested organs.

The following species dominated on the infested organs of 10 evergreen rhododendron taxa: $A$. alternata, P. sydowiana (syn. Pestalotia sydowiana), T. truncata (syn. Pestalotia truncata) and E. nigrum, which has been confirmed in other studies concerning the health of leaves, buds and flowers [1, 5, 13-15, 17-20]. Farr et al. [14] have written about the prevalence of $P$. sydowiana on the leaves and stems of Rhododendrons, e.g., in Chile, China and Canada.

Necrotrophs were diagnosed on infected evergreen Rhododendron organs from the following genera: Alternaria, Pestalotiopsis, Epicoccum and Sordaria; pathogens were from the genera: Botrytis, Fusarium and Gibberella; saprophytes were from the genera: Aspergillus, Boeremia, Chaetomium, Cladosporium, Coleophoma, Humicola, Mortierella, Paraphoma,
Penicillium, Phoma, Trichoderma and Umbelopsis, proving that the leaves were a source of infection for forming buds in autumn [5, 13, 14, 16, 20-22].

These results confirm the pathogenicity and aggressiveness, which many of these fungi have for flowers buds and petals as well as the ability to contaminate seeds. According to Płażek [23], necrotrophic pathogens kill the plant using toxins and then feed on dead tissue, which is overgrown by the network of mycelium. In this study, necrosis on the covering tissue of flowers buds was visible in the three years of research in the growing season and preceded the dying of flowers buds. The necrosis of buds formed as a symptom of hypersensitivity-hypersensitive response (HR), which is one of the main defense mechanisms that are activated by the plant during pathogen attack [4, 23].

In the discussion on the health of rhododendrons, the importance of phyllosphere is emphasized, where competition between pathogens and saprotrophs results in the dieback of affected organs. In the present study, there was no bud infection by Pycnostysanus azaleae, what is mentioned by Czekalski and Frużyńska-Jóźwiak [1] and Garibaldi et al. [16]. The following pathogens were not found on newly grown Rhododendron hybrids: Exobasidium vaccinii, Microsphaera penicillata, Colletotrichum gloeosporioides, Chrysomyxa spp., Pucciniastrum vaccinii, Puccinia rhododendri, Septoria azaleae, Cercospora handelii, Phyllosticta cunninghamii, Ovulinia azalea and Briosia azaleae; these pathogens are mentioned in numerous scientific description [8-10, $14,15,19,24]$.

Analyzing the number of micromycetes on the infested organs of different Rhododendrons taxa should take into account their sporulation, because a weak plant can keep its vital functions but it is not able to prevent the formation of the generative stage of parasite or conidia. Therefore, it appears that in the assessment of the susceptibility to colonization by micromycetes of Rhododendron taxa, mainly the size of colony should be taken into account $[5,13]$.

Because of the documented existence of numerous micromycetes colonies and species on the flower buds 
and petals of the taxa $R$. aureum $\times R$. brachycarpum and $R$. aureum $\times R$. yakushimanum "Koichiro Wada", resulting in dieback, these hybrids can be considered to have little use in selective breeding research. Hybrids of $R$. brachycarpum $\times R$. brachycarpum and R. yakushimanum "Koichiro Wada" $\times R$. catawbiense "Catherine van Tol" also proved to have little use, because their flower buds were colonized by the largest number of colonies and species of micromycetes communities. The seeds of $R$. purdomii $\times R$. yakushimanum "Koichiro Wada", R. aureum $\times R$. yakushimanum "Koichiro Wada" and $R$. yakushimanum "Koichiro Wada" $\times R$. brachycarpum were contaminated by numerous micromycetes colonies, which is associated with poor germination. The most useful Rhododendron hybrid for breeding was found to be seeds derived from $R$. aureum $\times R$. brachycarpum, $R$. brachycarpum $\times R$. brachycarpum and $R$. purdomii.

\section{Conclusions}

The low susceptibility of newly grown taxa $R$. brachycarpum $\times R$. purdomii, $R$. purdomii $\times R$. yakushimanum and $R$. purdomii species to the infection by micromycetes confirms their usefulness in further breeding work.

The propagating work should also be concentrated on the hybrids $R$. aureum $\times R$. catawbiense "Catherine van Tol", $R$. brachycarpum $\times R$. brachycarpum and $R$. brachycarpum $\times R$. purdomii, which showed variable quantitative parameters of micromycetes, concerning infected organs.

The present study confirmed the diversity of the micromycetes species that inhabit the infested Rhododendron buds, flowers and seeds of newly grown evergreen Rhododendron hybrids and compared their infection by micromycetes with the suitability for further breeding work.

\section{Acknowledgments}

Research was supported by the Polish Ministry of Science and Higher Education as part of the statutory activities of the Department of Plant Protection, University of Agriculture in Krakow.

\section{References}

[1] Czekalski, M., and Frużyńska-Jóźwiak, D. 1995. "Flower Bud Blast Caused by Fungus Pycnostysanus azaleae (Pech) Mason.” Erica Polonica 6: 7-14. (in Polish)

[2] Werner, M., Czekalski, M., and Frużyńska-Jóźwiak, D. 1996. "Petal Blight on Rhododendrons in Poland." Erica Polonica 7: 52-5. (in Polish)

[3] Muras, P. 2005. "Producing F1 Hybrids of the Genus Rhododendron L. and Assessing Their Morphological Characteristics and Frost Resistance." Habilitation thesis, Scientific Papers of Agricultural Academy in Kraków. (in Polish)

[4] Bertetti, D., Gullino, M., and Garibaldi, A. 2007. "Susceptibility of Evergreen Azalea Cultivars to Anthracnose Caused by Colletotrichu acutatum." Hort. Technology 17 (4): 501-4.

[5] Kowalik, M. 2008. "Fungi and Fungi-Like Omycetes Isolated from Affected Leaves of Rhododendron." Acta Mycol. 43 (1): 21-7.

[6] Guba, E. F. 1961. Monograph of Monochaetia and Pestalotia. Cambridge: Harvard University Press, 256.

[7] Domsch, K. H., Gams, W., and Anderson, T. H. 1980. Compendium of Soil Fungi. London: Academic Press, 859.

[8] Sutton, B. C. 1980. The Coelomycetes: Fungi Imperfecti with Pycnidia, Acervuli and Stromata. England: Commonwealth Mycological Institute, 696.

[9] Sivanesan, A. 1984. The Bitunicate Ascomycetes and Their Anamorphs. Vaduz: Lubrecht and Cramer Ltd., 700.

[10] Ellis, M. B., and Ellis, J. P. 1987. Microfungi on Land Plants: An Identification Handbook. London: Croom Helm, 818.

[11] Kirk, P. M., Cannon, P. F., Minster, D. W., and Stalpers, J. A. 2008. Ainsworth and Bisby's Dictionary of the Fungi, 10th ed.. Wallingford: CABI.

[12] Index Fungorum. 2015. Accessed January 27, 2015. www.indexfungorum.org.

[13] Kowalik, M. 2013. "Diversity of Fungi Colonizing and Damaging Leaves of Pontic Azalea Azalea pontica." Acta Mycol. 48 (2): 227-36.

[14] Farr, D. F., Esteban, H. B., and Palm, M. E. 1996. Fungi on Rhododendron: A World Reference. Boone, USA: Parkway Publishers Inc., 195.

[15] Werner, M., Frużyńska-Jóźwiak, D., and Czekalski, M. 1998. "Mycological Analysis of the Not Blooming Rhododendron Inflorences." Erica Polonica 9: 60-3. (in Polish)

[16] Garibaldi, A., Gilardi, G., Bertetti, D., and Gullino, M. L. 2002. "First Report of Pycnostysanus azaleae on Rhododendron in Italy." Plant Disease 86 (5): 560-3.

[17] Żołna, M., Kierpiec, B., and Kowalik, M. 2012. "Pathogens Decreasing Decorative Values of Rhododendron (Rhododendron L.) Flowers." Prog. Plant Prot. 52 (4): 1074-7. (in Polish) 
[18] Kita, W., and Mazurek, J. 2003. "Species Composition of Phyllosphere of Rhododendrons in Botanical Garden in Wrocław and Arboretum Wojsławice." Erica Polonica 14: 25-36. (in Polish)

[19] Kowalik, M. 2009. "Biodiversity of Fungi Occurring in Phyllosphere of Evergreen Rhododendron (Rhododendron L.)." Zeszyty Problemowe Postępów Nauk Rolnniczych 539: 341-8. (in Polish)

[20] Kowalik, M., Muras, P., Żołna, M., and Kierpiec, B. 2010. "Fungi Isolated from Necrotic Spots on the Leaves of Evergreen Rhododendron (Rhododendron L.)." Zesz. Probl. Post. Nauk Roln. 554: 49-55. (in Polish)

[21] Kowalik, M., Kierpiec, B., Bonio, J., and Żołna, M. 2011.
"Fungi Inhabiting Spots and Necroses on the Leaves of Azalea (Rhododendron) in the Botanical Garden of the Jagiellonian University." Phytopathologia 62: 41-8.

[22] Kowalik, M., Kierpiec, B., and Żołna, M. 2012. "Fungi Living at the Fallen Leaves of Rhododendron and Azalea (Rhododendron L.)." Acta Sci. Pol.-Hortorum Cultus 11 (2): 161-6.

[23] Płażek, A. 2011. Plant Pathophysiology. Krakow: Publishing House of the University of Agriculture in Krakow, 139. (in Polish)

[24] Łabanowski, G., Orlikowski, L., Soika, G., Wojdyła, A., and Korbin, M. 2001. Plant Protection of Ericaceae. Kraków: Plantpress Sp. z o.o., 113. (in Polish) 